\title{
Infinitely Many Solutions for a Class of Fractional Boundary Value Problems with Nonsmooth Potential
}

\author{
Kaimin Teng \\ Department of Mathematics, Taiyuan University of Technology, Taiyuan, Shanxi 030024, China \\ Correspondence should be addressed to Kaimin Teng; tengkaimin2013@163.com
}

Received 4 June 2013; Accepted 8 August 2013

Academic Editor: Salvatore A. Marano

Copyright (C) 2013 Kaimin Teng. This is an open access article distributed under the Creative Commons Attribution License, which permits unrestricted use, distribution, and reproduction in any medium, provided the original work is properly cited.

We establish the existence of infinitely many solutions for a class of fractional boundary value problems with nonsmooth potential. The technical approach is mainly based on a result of infinitely many critical points for locally Lipschitz functions.

\section{Introduction}

In the present paper, we are concerned with the existence of infinitely many solutions for a class of fractional boundary value problems with the following form:

$$
\begin{array}{r}
{ }_{t} D_{T}^{\alpha}\left({ }_{0} D_{t}^{\alpha} u(t)\right) \in \lambda \partial F(t, u(t)) \quad \text { a.e. } t \in[0, T], \\
u(0)=u(T)=0,
\end{array}
$$

where $\lambda \in \mathbb{R}$ is a parameter, and ${ }_{t} D_{T}^{\alpha}$ and ${ }_{0} D_{t}^{\alpha}$ are the left and right Riemann-Liouville fractional derivatives of order $0<$ $\alpha \leq 1$, respectively. $F:[0, T] \times \mathbb{R}^{N} \rightarrow \mathbb{R}$ is a given function satisfying some assumptions, and $\partial F(t, \cdot)$ is the generalized gradient in the sense of Clarke [1].

In particular, if $\alpha=1$, then problem (1) is reduced to the standard second-order boundary value problem

$$
\begin{array}{r}
\ddot{u}(t) \in \lambda \partial F(t, u(t)) \quad \text { a.e. } t \in[0, T], \\
u(0)=u(T)=0 .
\end{array}
$$

There are many excellent results that have been worked out on the existence of solutions for second-order BVP (we refer the reader to see $[2,3]$ and the references therein).

Recently, fractional differential equations and inclusions have attracted lots of people's interests because of their applications in viscoelasticity, electrochemistry, control, porous media, and so forth. The existence and multiplicity of solutions for BVP of fractional differential equations and inclusions have been established by some fixed-point theorems; we refer the readers to see [4-7].

This paper is motivated by the recent papers [8] where several existence results concerning problem (1) under the smooth case are obtained by using variational methods. In their papers, the authors define a suitable space and find a variational functional for fractional differential equations with Dirichlet boundary conditions. The aim of the present paper is to establish the existence of infinitely many solutions for problem (1) by using a critical points theorem according to Bonanno and Bisci [9].

It is interesting that the existence of infinitely many solutions for differential equations can be established without the symmetry assumption. Recently, Bonanno and Bisci in [9] established a precise version of the infinitely many critical points theorem of Marano and Motreanu [10] which extended the results of Ricceri in [11] for the nondifferentiable functionals. In applying the theorem, we need to assume some appropriate oscillating behavior of the nonlinear term either at infinity or at zero. This methodology has been usefully used in obtaining the existence of multiple results for different kinds of problems, such as p-Laplacian problem [12], quasilinear elliptic system [13, 14], discrete BVP [15], double Sturm-Liouville problem [16], and elliptic problems with variable exponent [17]. By using this methodology, to the best of our knowledge, it seems that no similar results are obtained in the literature for fractional BVP. Therefore, the purpose 
of our paper is to establish the existence of infinitely many solutions for problem (1) by using this type of methodology.

Our main results are stated as follows. For this matter, put

$$
\begin{gathered}
A=\liminf _{\xi \rightarrow+\infty} \frac{\int_{0}^{T} \max _{|x| \leq \xi} F(t, x) d t}{\xi^{2}} \\
B=\limsup _{|x| \rightarrow+\infty} \frac{\int_{T / 4}^{3 T / 4} F(t, x) d t}{|x|^{2}}, \\
C(T, \alpha)=\int_{0}^{T / 4} t^{2-2 \alpha} d t+\int_{T / 4}^{3 T / 4}\left[t^{1-\alpha}-\left(t-\frac{T}{4}\right)^{1-\alpha}\right]^{2} d t \\
+\int_{3 T / 4}^{T}\left[t^{1-\alpha}-\left(t-\frac{T}{4}\right)^{1-\alpha}+\left(t-\frac{3 T}{4}\right)^{1-\alpha}\right]^{2} d t .
\end{gathered}
$$

Our first main result is the following theorem.

Theorem 1. Suppose $F(t, x)$ satisfies the following conditions.

$\left(F_{0}\right)$ For all $x \in \mathbb{R}^{N}$, the function $t \rightarrow F(t, x)$ is measurable.

$\left(F_{1}\right)$ For almost all $t \in[0, T]$, the function $x \rightarrow F(t, x)$ is locally Lipschitz and $F(t, 0)=0$.

$\left(F_{2}\right)$ There exist $a, b \in L^{1}\left([0, T], \mathbb{R}^{+}\right)$such that $\left|x^{*}\right| \leq a(t)+$ $b(t)|x|^{r-1}$ with $r \in[1,+\infty)$ for all $x^{*} \in \partial F(t, x)$, all $x \in \mathbb{R}^{N}$, and almost all $t \in[0, T]$.

$\left(F_{3}\right) F(t, x) \geq 0$ for almost all $t \in[0, T]$ and all $x \in \mathbb{R}^{N}$.

$\left(F_{4}\right) A<\kappa B$, where $\kappa=(2 \alpha-1) T^{2} \Gamma^{2}(2-\alpha) \Gamma^{2}(\alpha) /$ $32 T^{2 \alpha-1} C(T, \alpha)$.

Then, for each $\lambda \in\left(16 C(T, \alpha) / B T^{2} \Gamma^{2}(2-\alpha),(2 \alpha-\right.$ 1) $\left.\Gamma^{2}(\alpha) / 2 A T^{2 \alpha-1}\right)$, problem (1) admits a sequence of solutions which is unbounded in $X$.

Next, we present the other main result. First, put

$$
\begin{gathered}
A_{1}=\liminf _{\xi \rightarrow 0^{+}} \frac{\int_{0}^{T} \max _{|x| \leq \xi} F(t, x) d t}{\xi^{2}}, \\
B_{1}=\limsup _{|x| \rightarrow 0^{+}} \frac{\int_{T / 4}^{3 T / 4} F(t, x) d t}{|x|^{2}} .
\end{gathered}
$$

Theorem 2. Suppose $F(t, x)$ satisfies the conditions $\left(F_{0}\right)-\left(F_{3}\right)$, and

$\left(F_{5}\right) A_{1}<\kappa B_{1}$, where $\kappa=(2 \alpha-1) T^{2} \Gamma^{2}(2-\alpha) \Gamma^{2}(\alpha) /$ $32 T^{2 \alpha-1} C(T, \alpha)$.

Then, for each $\lambda \in\left(16 C(T, \alpha) / B_{1} T^{2} \Gamma^{2}(2-\alpha),(2 \alpha-\right.$ 1) $\left.\Gamma^{2}(\alpha) / 2 A_{1} T^{2 \alpha-1}\right)$, problem (1) admits a sequence of pairwise distinct solutions which strongly converges to zero in $X$.

In order to prove Theorems 1 and 2, we recall the critical point theorem in [9] here for the readers' convenience.
Theorem 3. Let $X$ be a reflexive real Banach space, and let $\Phi, \Psi: X \rightarrow \mathbb{R}$ be two Lipschitz functions such that $\Phi$ is sequentially weakly lower semicontinuous and coercive and $\Psi$ is sequentially weakly upper semicontinuous. For every $r>$ $\inf _{X} \Phi$, one puts

$$
\begin{gathered}
\varphi(r)=\inf _{u \in \Phi^{-1}((-\infty, r))} \frac{\sup _{v \in \Phi^{-1}((-\infty, r))} \Psi(v)-\Psi(u)}{r-\Phi(u)}, \\
\gamma=\liminf _{r \rightarrow+\infty} \varphi(r), \quad \delta=\liminf _{r \rightarrow\left(\inf _{X} \Phi\right)^{+}} \varphi(r) .
\end{gathered}
$$

Then,

(a) if $\gamma<+\infty$, for each $\lambda \in(0,1 / \gamma)$, the following alternative holds: either

(i) $I_{\lambda}=\Phi-\lambda \Psi$ possesses a global minimum, or

(ii) there is a sequence $\left\{u_{n}\right\}$ of critical points (local minimum) of $I_{\lambda}$ such that $\lim _{n \rightarrow \infty} \Phi\left(u_{n}\right)=+\infty$.

(b) If $\delta<+\infty$, for each $\lambda \in(0,1 / \delta)$, the following alternative holds: either

(i) there is a global minimum of $\Phi$ which is a local minimum of $I_{\lambda}$, or

(ii) there is a sequence $\left\{u_{n}\right\}$ of pairwise distinct critical points (local minimum) of $I_{\lambda}$ with $\lim _{n \rightarrow \infty} \Phi\left(u_{n}\right)=\inf _{X} \Phi$, which weakly converges to a global minimum of $\Phi$.

The present paper is organized as follows. In Section 2 we present some basic definitions and facts from the nonsmooth analysis theory, and we prove a variational principle for problem (1). Section 3 is devoted to proving Theorems 1 and 2.

\section{Preliminaries}

2.1. Nonsmooth Analysis. Let $X$ be a real Banach space and $X^{*}$ its conjugate space; we denote by $\|\cdot\|$ and $\langle\cdot, \cdot\rangle$, respectively, the norm and the duality pairing between $X^{*}$ and $X$.

For a locally Lipschitz function $\varphi: X \rightarrow \mathbb{R}$, we define the generalized directional derivative of $\varphi$ at point $u$ in the direction $h \in E$ as follows:

$$
\varphi^{0}(u ; h)=\limsup _{v \rightarrow 0, s \downarrow 0} \frac{\varphi(u+v+s h)-\varphi(u+v)}{s} .
$$

The generalized gradient of a locally Lipschitz function $\varphi$ at the point $u$, denoted by $\partial \varphi(u)$, is the set $\partial \varphi(u)=\{w \in$ $\left.E^{*}:\langle w, v\rangle \leq \varphi^{0}(u ; v), \forall v \in X\right\}$. If $\varphi \in C^{1}(X)$, then $\partial \varphi(u)=$ $\left\{\varphi^{\prime}(u)\right\}$ for all $u \in X$.

A point $u \in X$ is said to be a critical point of a locally Lipschitz function $\varphi: X \rightarrow \mathbb{R}$ if $0 \in \partial \varphi(u)$. Clearly, if $u$ is a minimum of a locally Lipschitz function $\varphi$, then $0 \in \partial \varphi(u)$; that is, $u$ is a critical point of $\varphi$. 
2.2. Fractional Derivative Space. Throughout this paper, we denote the norm of the space $L^{p}\left([0, T], \mathbb{R}^{N}\right)$ for $1 \leq p \leq+\infty$ as $\|u\|_{L^{p}}=\left(\int_{0}^{T}|u(t)|^{p} d t\right)^{1 / p}$ and $\|u\|_{\infty}=\max _{u \in[0, T]}|u(t)|$.

Definition 4. Let $0<\alpha \leq 1$ and $1<p<\infty$. The fractional derivative space $E_{0}^{\alpha, p}$ is defined by the closure of $C_{0}^{\infty}\left([0, T], \mathbb{R}^{N}\right)$ with respect to the norm

$$
\begin{array}{r}
\|u\|_{\alpha, p}=\left(\int_{0}^{T}|u(t)|^{p} d t+\int_{0}^{T}\left|{ }_{0}^{c} D_{t}^{\alpha} u(t)\right|^{p} d t\right)^{1 / p}, \\
\forall u \in E_{0}^{\alpha, p} .
\end{array}
$$

Remark 5. It is obvious that this fractional derivative space $E_{0}^{\alpha, p}$ is the space of functions $u \in L^{p}\left([0, T], \mathbb{R}^{N}\right)$ having an $\alpha$-order Caputo fractional derivative ${ }_{0}^{c} D_{t}^{\alpha} u \in L^{p}\left([0, T], \mathbb{R}^{N}\right)$ and $u(0)=u(T)=0$.

The properties of the fractional derivative spaces $E_{0}^{\alpha, p}$ are listed as the following lemma.

Lemma 6 (see [8]). Let $0<\alpha \leq 1$ and $1<p<\infty$.

(1) The fractional derivative spaces $E_{0}^{\alpha, p}$ are a reflexive and separable Banach space.

(2) If $\alpha>1 / p$, for any $u \in E_{0}^{\alpha, p}$, one has ${ }_{0} D_{t}^{-\alpha}\left({ }_{0} D_{t}^{\alpha} u(t)\right)=$ $u(t)$, for any $t \in[0, T]$.

(3) If $1-\alpha \geq 1 / p$ or $\alpha>1 / p$, one has $E_{0}^{\alpha, p} \hookrightarrow$ $L^{p}\left([0, T], \mathbb{R}^{N}\right)$ is compact and

$$
\|u\|_{L^{p}} \leq \frac{T^{\alpha}}{\Gamma(\alpha+1)}\left\|_{0}^{c} D_{t}^{\alpha} u\right\|_{L^{p}}
$$

(4) Assume that $\alpha>1 / p$ and the sequence $\left\{u_{n}\right\}$ converges weakly to $u$ in $E_{0}^{\alpha, p}$; that is, $u_{n} \rightarrow u$. Then $\left\{u_{n}\right\}$ converges strongly to $u$ in $C\left([0, T], \mathbb{R}^{N}\right)$; that is, $\left\|u_{n}-u\right\|_{\infty} \rightarrow 0$, as $n \rightarrow+\infty$. Moreover, if $1 / p+$ $1 / q=1$, one has

$$
\|u\|_{\infty} \leq \frac{T^{\alpha-1 / p}}{\Gamma(\alpha)((\alpha-1) q+1)^{1 / q}}\left\|_{0}^{c} D_{t}^{\alpha} u\right\|_{L^{p}}
$$

According to (8), we can consider $E_{0}^{\alpha, p}$ with respect to the following norm:

$$
\|u\|_{\alpha, p}=\left\|{ }_{0}^{c} D_{t}^{\alpha} u\right\|_{L^{p}}=\left(\int_{0}^{T}\left|{ }_{0}^{c} D_{t}^{\alpha} u(t)\right|^{p} d t\right)^{1 / p} .
$$

In this paper, the work space for problem (1) is $E_{0}^{\alpha, 2}:=E^{\alpha}$. The space $E^{\alpha}$ is a Hilbert space with respect to the norm $\|u\|=$ $\|u\|_{\alpha, 2}$ given by (10), and the corresponding inner product is defined by the following:

$$
\langle u, v\rangle=\int_{0}^{T}\left({ }_{0}^{c} D_{t}^{\alpha} u(t),{ }_{0}^{c} D_{t}^{\alpha} v(t)\right) d t
$$

2.3. Variational Framework. We first give the definition for the solution of problem (1).

Definition 7. A function $u:[0, T] \rightarrow \mathbb{R}^{N}$ is called a solution of problem (1) if ${ }_{t} D_{T}^{\alpha-1}\left({ }_{0} D_{t}^{\alpha} u(t)\right)$ and ${ }_{0} D_{t}^{\alpha-1} u(t)$ are derivatives for almost all $t \in[0, T]$ and $u$ satisfies (1).

The functional $I_{\lambda}: E^{\alpha} \rightarrow \mathbb{R}$ corresponding to problem (1) is defined by the following:

$$
I_{\lambda}(u)=\frac{1}{2} \int_{0}^{T}\left|{ }_{0} D_{t}^{\alpha} u(t)\right|^{2} d t-\lambda \int_{0}^{T} F(t, u(t)) d t .
$$

By the conditions $\left(F_{0}\right)-\left(F_{2}\right)$, it is easy to check that $I_{\lambda}$ is locally Lipschitz on $E^{\alpha}$. Moreover, we can get the variational principle as follows.

Proposition 8. Every critical point $u \in E^{\alpha}$ of $I_{\lambda}$ is a solution of problem (1).

Proof. We assume that $u \in E^{\alpha}$ is a critical point of $I_{\lambda}$; that is $0 \in \partial I_{\lambda}(u)$; then,

$$
\int_{0}^{T}\left({ }_{0} D_{t}^{\alpha} u(t),{ }_{0} D_{t}^{\alpha} v(t)\right) d t-\lambda \int_{0}^{T}(w(t), v(t)) d t=0
$$

$\forall v \in E^{\alpha}$

with some $w \in \partial F(t, u)$. Noting that $w \in L^{1}\left([0, T], \mathbb{R}^{N}\right)$, then ${ }_{t} D_{T}^{-\alpha} w \in L^{1}\left([0, T], \mathbb{R}^{N}\right)$. Let $z(t)={ }_{t} D_{T}^{-\alpha} w(t), t \in[0, T]$. Hence, by the formula of integration by parts for the left and right Riemann-Liouville fractional derivatives, we have the following:

$$
\begin{aligned}
\int_{0}^{T}\left(z(t),{ }_{0} D_{t}^{\alpha} v(t)\right) d t & =\int_{0}^{T}\left({ }_{t} D_{T}^{\alpha} z(t), v(t)\right) d t \\
& =\int_{0}^{T}\left({ }_{t} D_{T}^{\alpha}\left({ }_{t} D_{T}^{-\alpha} w(t)\right), v(t)\right) d t \\
& =\int_{0}^{T}(w(t), v(t)) d t .
\end{aligned}
$$

By (13), for every $v \in E^{\alpha}$ and hence for every $v \in C_{0}^{\infty}([0$, $T], \mathbb{R}^{N}$ ), we have

$$
\int_{0}^{T}\left({ }_{0} D_{t}^{\alpha} u(t)-\lambda z(t),{ }_{0} D_{t}^{\alpha} v(t)\right) d t=0 .
$$

Since $v \in C_{0}^{\infty}\left([0, T], \mathbb{R}^{N}\right)$, we have ${ }_{0} D_{t}^{\alpha} v(t)={ }_{0} D_{t}^{\alpha-1} v^{\prime}(t)$. By the formula of integration by parts for the left and right Riemann-Liouville fractional derivatives, we get

$$
\begin{array}{r}
\int_{0}^{T}\left({ }_{t} D_{T}^{\alpha-1}\left({ }_{0} D_{t}^{\alpha} u(t)-\lambda z(t)\right), v^{\prime}(t)\right) d t=0, \\
\forall v \in C_{0}^{\infty}\left([0, T], \mathbb{R}^{N}\right) .
\end{array}
$$

Since ${ }_{t} D_{T}^{\alpha-1}\left({ }_{0} D_{t}^{\alpha} u-\lambda z\right) \in L^{1}\left([0, T], \mathbb{R}^{N}\right)$, the standard Fourier series theory implies that

$$
{ }_{t} D_{T}^{\alpha-1}\left({ }_{0} D_{t}^{\alpha} u(t)-\lambda z(t)\right)=C_{0}, \quad \text { for almost all } t \in[0, T]
$$


for some constant $C_{0} \in \mathbb{R}^{N}$. Using the properties of the left and right Riemann-Liouville fractional derivatives, we have

$$
{ }_{0} D_{t}^{\alpha} u(t)=\lambda z(t)+C(T-t)^{\alpha-1}, \quad \text { a.e. } t \in[0, T] \text {. }
$$

Since $w(t) \in L^{1}\left([0, T], \mathbb{R}^{N}\right)$, we can identify the equivalence class ${ }_{t} D_{T}^{\alpha-1}\left({ }_{0} D_{t}^{\alpha} u\right)$ and its continuous representant

$$
{ }_{t} D_{T}^{\alpha-1}\left({ }_{0} D_{t}^{\alpha} u(t)\right)=\lambda \int_{t}^{T} w(t) d t+C, \quad \text { for } t \in[0, T] .
$$

Firstly, we notice that ${ }_{0} D_{t}^{\alpha-1} u(t)$ is derivative for almost every $t \in[0, T]$ and $\left({ }_{0} D_{t}^{\alpha-1} u(t)\right)^{\prime}={ }_{0} D_{t}^{\alpha} u(t) \in L^{2}\left([0, T], \mathbb{R}^{N}\right)$ as $u \in$ $E^{\alpha}$. On the other hand, ${ }_{t} D_{T}^{\alpha-1}\left({ }_{0} D_{t}^{\alpha} u(t)\right)$ is derivative a.e. on $[0, T]$ and $\left({ }_{t} D_{T}^{\alpha-1}\left({ }_{0} D_{t}^{\alpha} u(t)\right)\right)^{\prime} \in L^{1}\left([0, T], \mathbb{R}^{N}\right)$.

By (19), we have

$$
\begin{array}{r}
{ }_{t} D_{T}^{\alpha}\left({ }_{0} D_{t}^{\alpha} u(t)\right)=-\left({ }_{t} D_{T}^{\alpha-1}\left({ }_{0} D_{t}^{\alpha} u(t)\right)\right)^{\prime}=\lambda w(t), \\
\text { a.e. } t \in[0, T] .
\end{array}
$$

Moreover, $u \in E^{\alpha}$ implies that $u(0)=u(T)=0$. The proof is completed.

\section{Proof of Main Results}

Throughout this section, for $u \in X:=E^{\alpha}$, we denote $I_{\lambda}(u)=$ $\Phi(u)-\lambda \Psi(u)$, where $\lambda \in\left(16 C(T, \alpha) / B T^{2} \Gamma^{2}(2-\alpha),(2 \alpha-\right.$ 1) $\left.\Gamma^{2}(\alpha) / 2 A T^{2 \alpha-1}\right)$, and

$$
\Phi(u)=\frac{1}{2}\|u\|^{2}, \quad \Psi(u)=\int_{0}^{T} F(t, u(t)) d t
$$

Clearly, $\Phi$ is Gâteaux differentiable and sequentially weakly lower semicontinuous and coercive; $\Psi$ is locally Lipschitz continuous on $X$; by standard argument, $\Psi$ is sequentially weakly continuous. We denote

$$
\begin{gathered}
A:=\liminf _{\xi \rightarrow+\infty} \frac{\int_{0}^{T} \max _{|x| \leq \xi} F(t, x) d t}{\xi^{2}}, \\
B:=\limsup _{|x| \rightarrow+\infty} \frac{\int_{0}^{T} F(t, x) d t}{|x|^{2}} .
\end{gathered}
$$

Proof of Theorem 1. First, we verify that $\gamma<+\infty$. By $\left(F_{4}\right)$, let $\left\{a_{n}\right\}$ be a real sequence such that $\lim _{n \rightarrow \infty} a_{n}=+\infty$ and

$$
\lim _{n \rightarrow \infty} \frac{\int_{0}^{T} \max _{|x| \leq a_{n}} F(t, x) d t}{a_{n}^{2}}=A .
$$

Put $r_{n}=\left((2 \alpha-1) \Gamma^{2}(\alpha) / 2 T^{2 \alpha-1}\right) a_{n}^{2}$ for all $n \in \mathbb{N}$. From (9), one has $\|u\|_{\infty} \leq a_{n}$ for all $u \in X$ such that $\|u\|^{2} \leq 2 r_{n}$. Take into account that $\left\|u_{0}\right\|=0$ and $\int_{0}^{T} F(t, 0) d t=0$, where $u_{0}(t)=0$ for all $t \in[0, T]$. For all $n \in \mathbb{N}$, we have

$$
\begin{aligned}
\varphi\left(r_{n}\right) & =\inf _{\|u\|^{2}<2 r_{n}} \frac{\sup _{\|v\|^{2} \leq 2 r_{n}} \int_{0}^{T} F(t, v(t)) d t-\int_{0}^{T} F(t, u(t)) d t}{r_{n}-\|u\|^{2} / 2} \\
& \leq \frac{\sup _{\|v\|^{2} \leq 2 r_{n}} \int_{0}^{T} F(t, v(t)) d t}{r_{n}} \\
& \leq \frac{2 T^{2 \alpha-1}}{(2 \alpha-1) \Gamma^{2}(\alpha)} \frac{\int_{0}^{T} \max _{|x| \leq a_{n}} F(t, x) d t}{a_{n}^{2}} .
\end{aligned}
$$

Since from assumption $\left(F_{4}\right)$ one has $A<+\infty$, then we have

$$
\gamma \leq \liminf _{n \rightarrow+\infty} \varphi\left(r_{n}\right) \leq \frac{2 T^{2 \alpha-1}}{(2 \alpha-1) \Gamma^{2}(\alpha)} A<+\infty .
$$

Now fix $\lambda \in\left(16 C(T, \alpha) / B T^{2} \Gamma^{2}(2-\alpha),(2 \alpha-1) \Gamma^{2}(\alpha) /\right.$ $\left.2 A T^{2 \alpha-1}\right)$. We claim that the functional $I_{\lambda}$ is unbounded from below. and

$\operatorname{By}\left(F_{4}\right)$, let $\left\{b_{n}\right\}$ be a sequence of $\mathbb{R}^{N}$ such that $\left|b_{n}\right| \rightarrow+\infty$

$$
\lim _{n \rightarrow+\infty} \frac{\int_{T / 4}^{3 T / 4} F\left(t, b_{n}\right) d t}{\left|b_{n}\right|^{2}}=B
$$

For each $n \in \mathbb{N}$, we define a sequence $\left\{w_{n}\right\}$ as follows:

$$
w_{n}(t)= \begin{cases}\frac{4 b_{n}}{T} t, & t \in\left[0, \frac{T}{4}\right), \\ b_{n}, & t \in\left[\frac{T}{4}, \frac{3 T}{4}\right], \\ \frac{4 b_{n}}{T}(T-t), & t \in\left(\frac{3 T}{4}, T\right] .\end{cases}
$$

It is easy to check that $w_{n}(0)=w_{n}(T)=0$ and $w_{n} \in L^{2}([0, T])$. Moreover, $w_{n}(t)$ is Lipschitz continuous on $[0, T]$, and hence $w_{n}(t)$ is absolutely continuous on $[0, T]$. By calculations, we get

$$
{ }_{0} D_{t}^{\alpha} w_{n}(t)=\left\{\begin{array}{c}
\frac{4 b_{n}}{T \Gamma(2-\alpha)} t^{1-\alpha}, \quad t \in\left[0, \frac{T}{4}\right), \\
\frac{4 b_{n}}{T \Gamma(2-\alpha)}\left[t^{1-\alpha}-\left(t-\frac{T}{4}\right)^{1-\alpha}\right] \\
t \in\left[\frac{T}{4}, \frac{3 T}{4}\right], \\
\left.+\left(t-\frac{3 T}{4}\right)^{1-\alpha}\right], \\
t \in\left(\frac{3 T}{4}, T\right] .
\end{array}\right.
$$


Obviously, ${ }_{0} D_{t}^{\alpha} w_{n}(t)$ is continuous on $[0, T]$ and

$$
\begin{aligned}
\int_{0}^{T}\left|{ }_{0} D_{t}^{\alpha} w_{n}(t)\right|^{2}= & \frac{16\left|b_{n}\right|^{2}}{T^{2} \Gamma^{2}(2-\alpha)} \\
& \times\left\{\int_{0}^{T / 4} t^{2-2 \alpha} d t\right. \\
& +\int_{T / 4}^{3 T / 4}\left[t^{1-\alpha}-\left(t-\frac{T}{4}\right)^{1-\alpha}\right]^{2} d t \\
& +\int_{3 T / 4}^{T}\left[t^{1-\alpha}-\left(t-\frac{T}{4}\right)^{1-\alpha}\right. \\
:= & \frac{16\left|b_{n}\right|^{2}}{T^{2} \Gamma^{2}(2-\alpha)} C(T, \alpha),
\end{aligned}
$$

where $C(T, \alpha)$ depends on $T$ and $\alpha$.

By condition $\left(F_{3}\right)$, we have

$$
\int_{0}^{T} F\left(t, w_{n}(t)\right) d t \geq \int_{T / 4}^{3 T / 4} F\left(t, b_{n}\right) d t
$$

for all $n \in \mathbb{N}$. Then,

$$
\begin{aligned}
I_{\lambda}\left(w_{n}\right)= & \Phi\left(w_{n}\right)-\lambda \Psi\left(w_{n}\right) \\
\leq & \frac{16\left|b_{n}\right|^{2}}{T^{2} \Gamma^{2}(2-\alpha)} C(T, \alpha) \\
& -\lambda \int_{T / 4}^{3 T / 4} F\left(t, b_{n}\right) d t
\end{aligned}
$$

for every $n \in \mathbb{N}$.

If $B<+\infty$, let $\varepsilon \in\left(16 C(T, \alpha) / \lambda B T^{2} \Gamma^{2}(2-\alpha), 1\right)$; by (26) there exists $N_{\varepsilon}>0$ such that

$$
\int_{T / 4}^{3 T / 4} F\left(t, b_{n}\right) d t \geq \varepsilon B\left|b_{n}\right|^{2}
$$

for all $n \geq N_{\varepsilon}$. Hence, by (31) and (32), we obtain

$$
I_{\lambda}\left(w_{n}\right) \leq\left[\frac{16}{T^{2} \Gamma^{2}(2-\alpha)} C(T, \alpha)-\lambda \varepsilon B\right]\left|b_{n}\right|^{2}
$$

for all $n \geq N_{\varepsilon}$. Choosing suitable $\varepsilon$, we have

$$
\lim _{n \rightarrow+\infty} I_{\lambda}\left(w_{n}\right)=-\infty
$$

On the other hand, if $B=+\infty$, we fix $M>16 C(T, \alpha) / \lambda T^{2} \Gamma^{2}$ $(2-\alpha)$, and again from (26) there exists $N_{M} \in \mathbb{N}$ such that

$$
\int_{T / 4}^{3 T / 4} F\left(t, b_{n}\right) d t \geq M\left|b_{n}\right|^{2}
$$

for all $n>N_{M}$. Therefore, from (31) and (35), we have

$$
I_{\lambda}\left(w_{n}\right) \leq\left[\frac{16}{T^{2} \Gamma^{2}(2-\alpha)} C(T, \alpha)-\lambda M\right]\left|b_{n}\right|^{2}
$$

for all $n>N_{M}$. From the choice of $M$, we have

$$
\lim _{n \rightarrow+\infty} I_{\lambda}\left(w_{n}\right)=-\infty
$$

Hence, our claim is proved.

Since all the assumptions of the case (a) of Theorem 3 are verified, for each $\lambda \in\left(16 C(T, \alpha) / B T^{2} \Gamma^{2}(2-\alpha),(2 \alpha-\right.$ 1) $\left.\Gamma^{2}(\alpha) / 2 A T^{2 \alpha-1}\right)$, the functional $I_{\lambda}$ admits an unbounded sequence of critical points. The conclusion follows from Proposition 8.

Proof of Theorem 2. The proof is the same as Theorem 1 by using the case (b) of Theorem 3 instead of the case (a).

Example 9. We give an example to illustrate Theorem 1. Set

$$
a_{n}=n ! n, \quad b_{n}=n !\left(n+\frac{1}{2}\right)
$$

for every $n \in \mathbb{N}$. Define the nonnegative function $F(t, x)$ : $[0, T] \times \mathbb{R}^{N} \rightarrow \mathbb{R}$ as follows:

$$
F(t, x)= \begin{cases}\left(|x|-\frac{n !}{2}\right)^{2} e^{-\left|1 /\left((|x|-n ! n-n ! / 2)^{2}-(n ! / 2)^{2}\right)\right|} \cos \frac{\pi}{2 T} t & \text { if } n ! n<|x|<(n+1) ! \\ 0, & \text { otherwise }\end{cases}
$$

Obviously, $F(t, x)$ satisfies the conditions $\left(F_{0}\right)-\left(F_{3}\right)$. Next, we show that $\left(F_{4}\right)$ is true. Indeed, by direct computation, we get

$$
\begin{gathered}
\lim _{n \rightarrow \infty} \frac{\int_{0}^{T} \max _{|x| \leq a_{n}} F(t, x) d t}{a_{n}^{2}}=0, \\
\lim _{n \rightarrow \infty} \frac{\int_{T / 4}^{3 T / 4} F\left(t, b_{n}\right) d t}{b_{n}^{2}}=\frac{\pi}{2 T}\left(\sin \frac{3 \pi}{8}-\sin \frac{\pi}{8}\right) .
\end{gathered}
$$

Hence, we see that

$$
\begin{aligned}
& A=\liminf _{\xi \rightarrow+\infty} \frac{\int_{0}^{T} \max _{|x| \leq \xi} F(t, x) d t}{\xi^{2}}=0, \\
& 0<B=\limsup _{|x| \rightarrow+\infty} \frac{\int_{T / 4}^{3 T / 4} F(t, x) d t}{|x|^{2}}<+\infty .
\end{aligned}
$$

Therefore, $\left(F_{4}\right)$ is verified.

\section{Acknowledgments}

The author is supported by the NSFC under Grant 11226117 and the Shanxi Province Science Foundation for Youths under Grant 2013021001-3. 


\section{References}

[1] F. H. Clarke, Optimization and Nonsmooth Analysis, Canadian Mathematical Society Series of Monographs and Advanced Texts, John Wiley \& Sons, New York, Ny, USA, 1983.

[2] S. Aizicovici, N. S. Papageorgiou, and V. Staicu, "Periodic solutions for second order differential inclusions with the scalar $p$ Laplacian," Journal of Mathematical Analysis and Applications, vol. 322, no. 2, pp. 913-929, 2006.

[3] L. Gasiński and N. S. Papageorgiou, Nonsmooth Critical Point Theory and Nonlinear Boundary Value Problems, vol. 8 of Series in Mathematical Analysis and Applications, Chapman \& Hall/CRC, Boca Raton, Fla, USA, 2005.

[4] R. P. Agarwal, M. Belmekki, and M. Benchohra, "A survey on semilinear differential equations and inclusions involving Riemann-Liouville fractional derivative," Advances in Difference Equations, vol. 2009, Article ID 981728, 47 pages, 2009.

[5] B. Ahmad and V. Otero-Espinar, "Existence of solutions for fractional differential inclusions with antiperiodic boundary conditions," Boundary Value Problems, vol. 2009, Article ID 625347, 11 pages, 2009.

[6] M. Benchohra, J. Henderson, S. K. Ntouyas, and A. Ouahab, "Existence results for fractional functional differential inclusions with infinite delay and applications to control theory," Fractional Calculus \& Applied Analysis, vol. 11, no. 1, pp. 35-56, 2008.

[7] V. Lakshmikantham and A. S. Vatsala, "Basic theory of fractional differential equations," Nonlinear Analysis. Theory, Methods \& Applications, vol. 69, no. 8, pp. 2677-2682, 2008.

[8] F. Jiao and Y. Zhou, "Existence results for fractional boundary value problem via critical point theory," International Journal of Bifurcation and Chaos in Applied Sciences and Engineering, vol. 22, no. 4, Article ID 1250086, 17 pages, 2012.

[9] G. Bonanno and G. M. Bisci, "Infinitely many solutions for a boundary value problem with discontinuous nonlinearities," Boundary Value Problems, vol. 2009, Article ID 670675, 20 pages, 2009.

[10] S. A. Marano and D. Motreanu, "Infinitely many critical points of non-differentiable functions and applications to a Neumanntype problem involving the $p$-Laplacian," Journal of Differential Equations, vol. 182, no. 1, pp. 108-120, 2002.

[11] B. Ricceri, "A general variational principle and some of its applications," Journal of Computational and Applied Mathematics, vol. 113, no. 1-2, pp. 401-410, 2000.

[12] G. Bonanno and G. Molica Bisci, "Infinitely many solutions for a Dirichlet problem involving the $p$-Laplacian," Proceedings of the Royal Society of Edinburgh A, vol. 140, no. 4, pp. 737-752, 2010.

[13] G. Bonanno, G. M. Bisci, and D. O’Regan, "Infinitely many weak solutions for a class of quasilinear elliptic systems," Mathematical and Computer Modelling, vol. 52, no. 1-2, pp. 152160,2010 .

[14] G. A. Afrouzi and A. Hadjian, "Infinitely many solutions for a class of Dirichlet quasilinear elliptic systems," Journal of Mathematical Analysis and Applications, vol. 393, no. 1, pp. 265272, 2012.

[15] G. Bonanno and P. Candito, "Infinitely many solutions for a class of discrete non-linear boundary value problems," Applicable Analysis, vol. 88, no. 4, pp. 605-616, 2009.

[16] G. D’Aguì, "Infinitely many solutions for a double SturmLiouville problem," Journal of Global Optimization, vol. 54, no. 3, pp. 619-625, 2012.
[17] G. D'Aguì and A. Sciammetta, "Infinitely many solutions to elliptic problems with variable exponent and nonhomogeneous Neumann conditions," Nonlinear Analysis. Theory, Methods \& Applications, vol. 75, no. 14, pp. 5612-5619, 2012. 


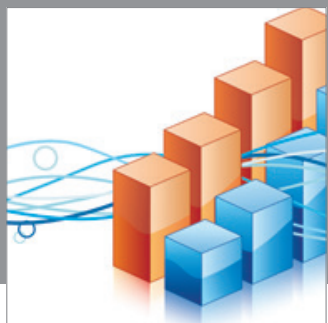

Advances in

Operations Research

mansans

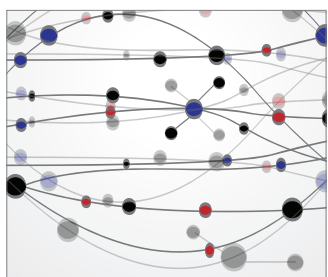

The Scientific World Journal
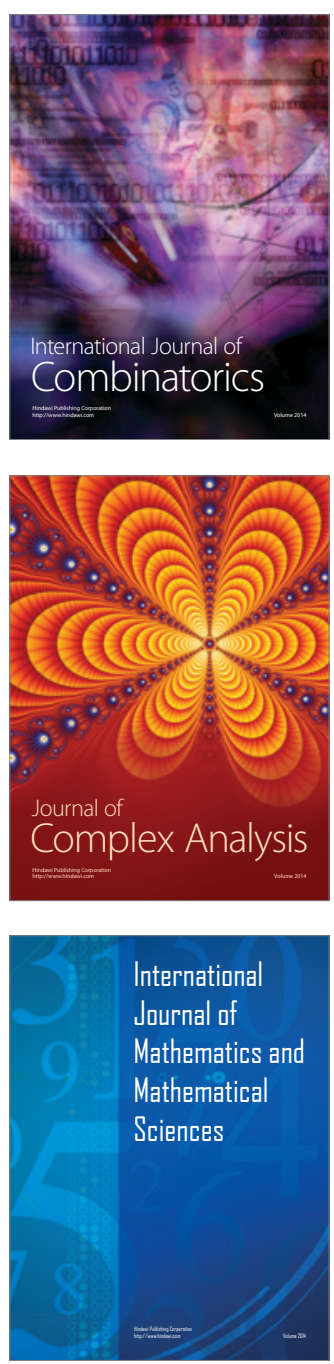
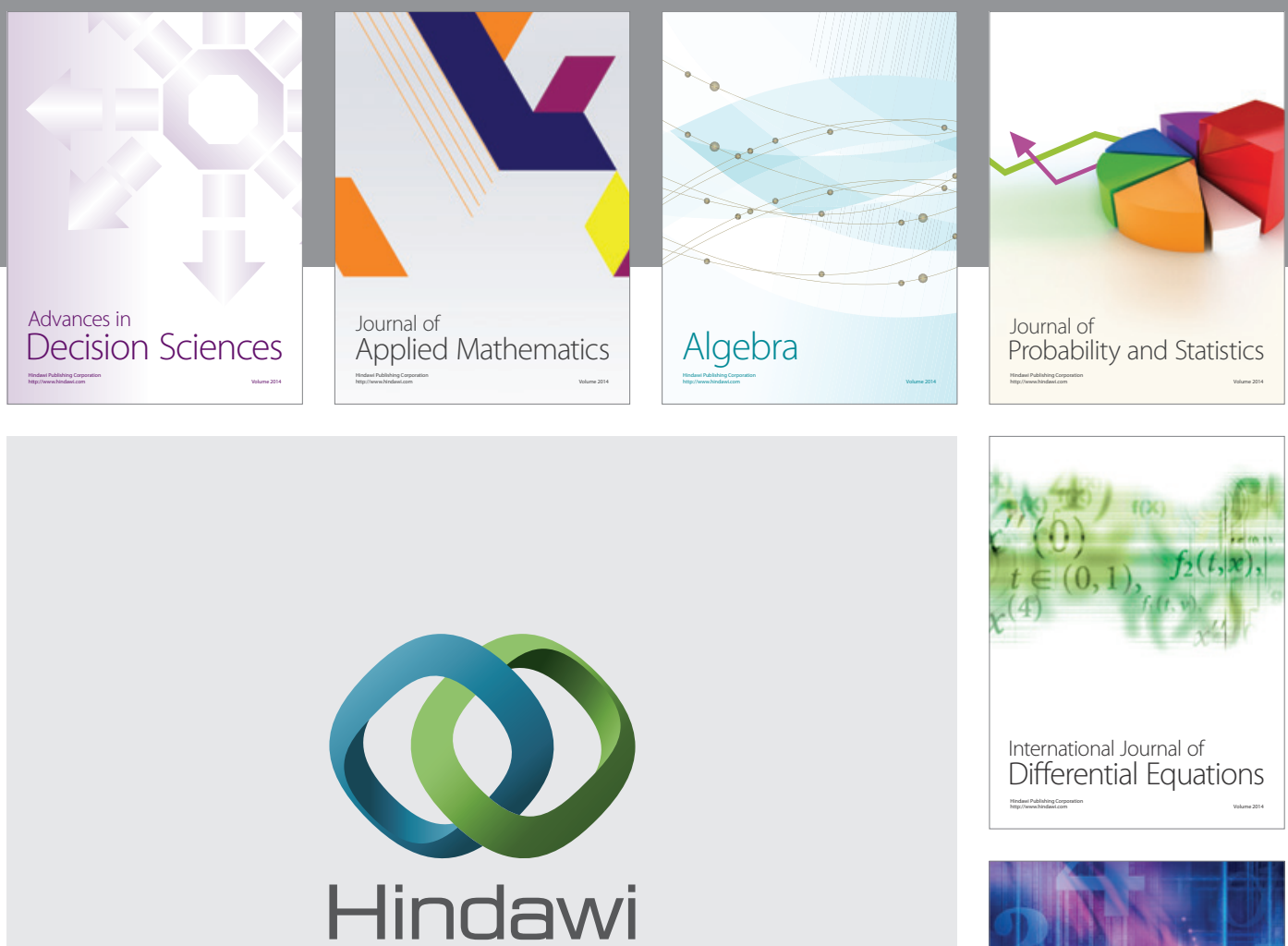

Submit your manuscripts at http://www.hindawi.com
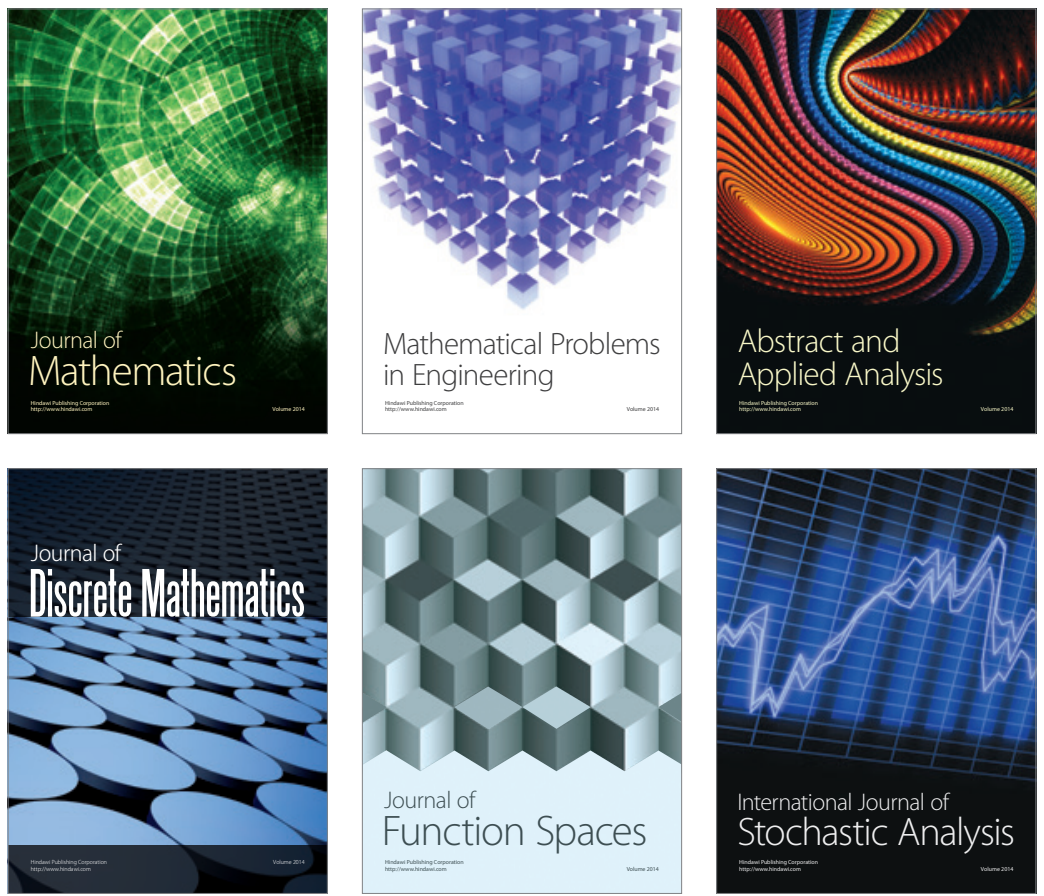

Journal of

Function Spaces

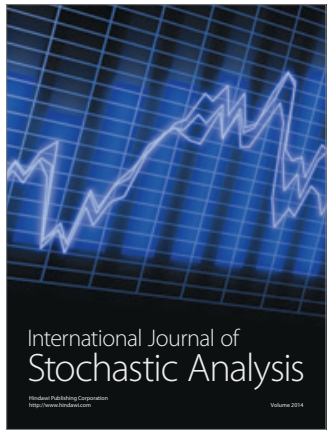

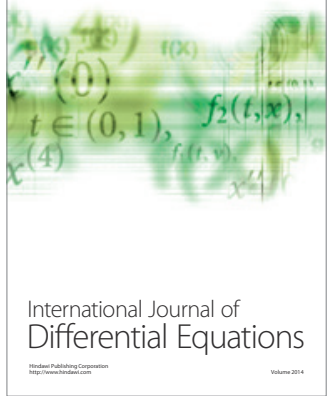
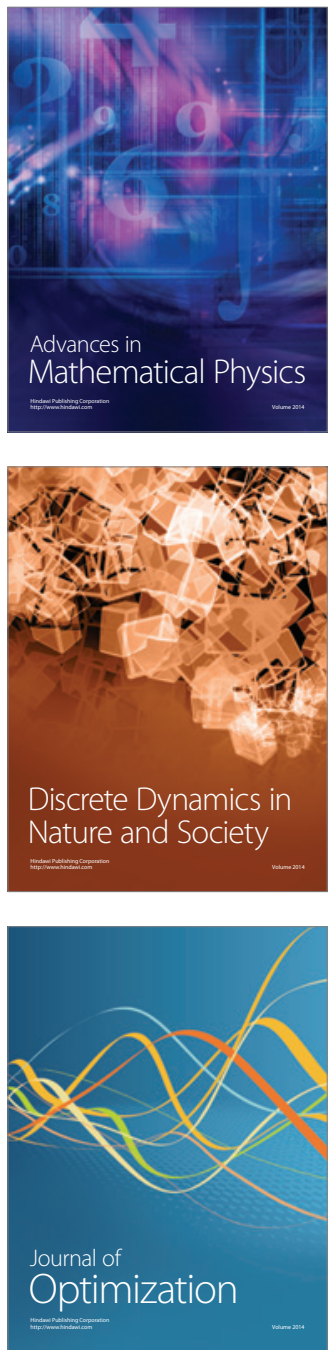